ERC-Leitlinien

Notfall Rettungsmed 2020 $23: 263-267$

https://doi.org/10.1007/s10049-020-00724-5

(c) European Resuscitation Council (ERC), German Resuscitation Council (GRC) 2020
P. Van de Voorde - L. Bossaert · S. Mentzelopoulos - M. T. Blom · K. Couper · J. Djakow · P. Druwé · G. Lilja · I. Lulic · V. Raffay · G. D. Perkins · K. G. Monsieurs European Resuscitation Council VZW, Niel, Belgien

\title{
Ethik der Reanimation und Entscheidungen am Lebensende
}

\section{COVID-19-Leitlinien des European Resuscitation Council}

Key Messages

- Alle „vorübergehenden“ Anpassungen an bestehende Leitlinien sollen im Kontext jedes einzelnen Gesundheitssystems interpretiert werden, unter Berücksichtigung der Verbreitung von COVID-19, der verfügbaren Ressourcen usw. Unser Wissen über COVID-19 ist immer noch begrenzt, und die Leitlinien müssen möglicherweise aktualisiert werden, sobald weitere Daten verfügbar sind.

- Die allgemeinen ethischen Grundsätze der Wiederbelebung bleiben gültig. Wenn möglich, soll „advanced care planning" (ACP - vorausschauende Behandlungsentscheidung; Patientenverfügung) in Betracht gezogen werden [1]. Dies kann im Zusammenhang mit der aktuellen COVID-19-Pandemie aufgrund der Wissenslücken, der Maßnahmen zur sozialen Distanzierung usw. besonders schwierig sein. Wir betrachten die kardiopulmonale Wiederbelebung (CPR) als eine Behandlung

Diese Leitlinie wurde am 24. April 2020 erstellt und unterliegt den sich weiterentwickelnden Kenntnissen und Erfahrungen über COVID-19. Da sich die Länder in verschiedenen Stadien der Pandemie befinden, kann es in der Praxis zu Abweichungen in einzelnen Ländern kommen.

Dieser Artikel ist eine Übersetzung durch den German Resuscitation Council der Covid-19 Leitlinien des ERC. Die Originalpublikation dieses Kapitels finden Sie hier https://www. erc.edu/sites/5714e77d5e615861f00f7d18/ content_entry5ea884fa4c84867335e4d1ff/ 5ea886c54c84867421e4d1fc/files/ERC covid19_pages_section7.pdf?1588257421 „unter Vorbehalt" und schlagen Kriterien für das Zurückhalten oder die Beendigung der Wiederbelebung vor. Die Umsetzung dieser Kriterien in einem Gesundheitssystem hängt vom lokalen Kontext ab (rechtlich, kulturell und organisatorisch).

- Behandlungsteams sollen für jeden einzelnen Patienten sorgfältig dessen Überlebenschancen und/oder "gutes" langfristiges Outcome sowie den erwarteten Ressourcenverbrauch bewerten. Da es sich hierbei nicht um eine statische Situation handelt, soll eine solche Bewertung regelmäßig überprüft werden. Wir raten davon ab, Kategorien oder „pauschale“ Kriterien (z. B. Altersschwellen) zu verwenden, um die „Berechtigung" eines Patienten festzulegen, bestimmte Ressourcen zu erhalten oder nicht zu erhalten.

- Die zentrale Herausforderung bei der Wiederbelebung während der COVID-19-Pandemie ist die Schwierigkeit, das Risiko für den Anwender und den potenziellen Nutzen für den Patienten zuverlässig auszubalancieren. Während medizinisches Personal sein Bestes für jeden einzelnen Patienten gibt, soll es sich zugleich seiner Verantwortung gegenüber Verwandten, Kollegen und der Gesellschaft bewusst sein. Angehörige von Gesundheitsberufen (einschließlich Ersthelfer) sollen bei allen Patienten mit bestätigtem oder vermutetem COVID-19 persönliche Schutzausrüstung (PSA) verwenden. Die Art der PSA soll auf Systemebe- ne im Verhältnis zum vermuteten Übertragungsrisiko definiert werden. Während das Vorgehen lokal an die aktuelle Realität der Pandemie angepasst werden kann, ist es, um einen exzessiven Anstieg von Mortalität und Morbidität durch den verzögerten Beginn der Herz-LungenWiederbelebung (HLW) zu vermeiden, unerlässlich, dass wir weiterhin eine von Leitstellendisponenten unterstützte CPR (Telefonreanimation) anbieten und Laienhelfer rekrutieren, ausbilden und/oder bei Kreislaufstillstand "first responder" senden.

\section{Einführung}

Die COVID-19(Coronavirus Disease 2019)-Pandemie stellt eine weltweite Krise dar, die in vielen Regionen zu erheblicher Morbidität und Mortalität führt. Das SARS-CoV-2(Severe Acute Respiratory Syndrome Coronavirus 2)Virus ist hochansteckend und ohne Immunität der Bevölkerung wesentlich tödlicher als die saisonale Influenza, insbesondere bei den am stärksten gefährdeten Menschen [2]. COVID-19 ist eine „neue“ Krankheit, und trotz vieler kürzlich veröffentlichter Studien ist unser Wissen darüber immer noch sehr begrenzt.

Es wurden viele damit verbundene $\mathrm{Ri}$ siken identifiziert, die das bereits angespannte Gesundheitssystem weiter unter Druck setzen und zu einer übermäßigen Mortalität führen können [3, 4]: 
- Wenn viele Menschen gleichzeitig erkranken, kann der Ressourcenbedarf die Ressourcenverfügbarkeit erheblich übersteigen. Dies umfasst u. a. Intensivpflegebetten, Beatmungsgeräte, Medikamente, Testmaterialien und persönliche Schutzausrüstung (PSA).

- Medizinisches Personal hat ein erhöhtes Risiko, sich mit COVID$19 \mathrm{zu}$ infizieren, was zusätzliche Herausforderungen für eine ausreichende Personalbesetzung für die direkte Patientenversorgung und die Hintergrundarbeit mit sich bringt.

- Störungen des Gesundheitssystems (aufgrund unzureichender Ressourcen, geringerer Bereitstellung auch von nicht COVID-19-bezogener Versorgung und v. a. übertriebener Angst) wirken sich auch auf die Versorgung von Patienten mit anderen akuten und chronischen medizinischen Problemen aus. Letztlich könnte dies zu einer höheren Morbidität und Mortalität führen als COVID-19 selbst [5].

In Anbetracht dessen hat die „ERC Ethics Writing Group“ (WG) einen klaren Bedarf an ethischen Leitlinien festgestellt. Wir sind uns darüber im Klaren, dass wichtige Änderungen der Leitlinien zur Wiederbelebung erhebliche und möglicherweise dauerhafte Auswirkungen auf das Patientenüberleben haben können.

Alle „vorübergehenden“ Anpassungen der bestehenden Leitlinien sollen immer im Kontext jedes Gesundheitssystems interpretiert werden und Faktoren wie die Prävalenz von COVID-19 in einer Region und die Gesamtauswirkung auf die vorhandenen Ressourcen berücksichtigen. Angesichts der begrenzten verfügbaren Evidenz sind die meisten der folgenden Aussagen das Ergebnis eines Expertenkonsenses. Sie basieren auf der jüngsten systematischen ILCORÜberprüfung des Risikos der Übertragung von COVID-19 auf Helfer während der Wiederbelebung, auf bestehenden Leitlinien anderer Fachgesellschaften und Berufsverbände sowie auf jüngsten, klinischen Studien, die meistens auf Beobachtungen beruhen [4, 6-12]. Auch indirekte Evidenz aus nicht kli- nischen Veröffentlichungen, wie z. B: über die Pathophysiologie, haben unsere endgültigen „Erkenntnisse“ beeinflusst.

\section{Gesundheitsorganisation während der COVID-19- Pandemie}

Basierend auf den Grundsätzen von Fürsorge, Gerechtigkeit und Gleichheit soll jeder einzelne Patient Zugang zum aktuellen Behandlungsstandard haben. Jedoch muss der Nutzen für den einzelnen Patienten mit dem für die gesamte Gesellschaft ausgeglichen werden. Insbesondere wenn der Bedarf an Gesundheitsressourcen die Kapazität übersteigt, kann dies bedeuten, die bestmögliche medizinische Versorgung für eine möglichst große Anzahl von Menschen anzustreben (Verteilungsgerechtigkeit; [12, 13]).

Während die Gesundheitssysteme im Wesentlichen bestrebt sein sollen, allen zu helfen, denen sie dienen, und darauf gut vorbereitet sein sollen, ist das Ausmaß der Krise so groß, dass es derzeit in manchen Regionen die vorhandenen Ressourcen überfordern könnte [2, 14]. Wenn ein klares Ungleichgewicht zwischen Ressourcenbedarf und verfügbarer Kapazität besteht, sollen Richtlinien für die Zuweisung und Verteilung von Ressourcen auf Systemebene (z. B. Regierung, nationale Behörden) und nicht von einzelnen Institutionen oder medizinischem Personal entwickelt werden.

Solche Richtlinien sollen sowohl von Angehörigen der Gesundheitsberufe mit unterschiedlichem Hintergrund als auch von Experten für medizinische Ethik, Recht, Wirtschaft und Soziologiekonsentiert werden. Besondere Aufmerksamkeit soll schutzbedürftigen Bevölkerungsgruppen gewidmet werden, die trotz eines höheren Risikos, zu erkranken, einem höheren Risiko einer „ungerechtfertigten Diskriminierung " ausgesetzt sind $[13,15,16]$. Die Effektivität jeder Maßnahme hängt vom Vertrauen der Bevölkerung in die Gesundheitsbehörden, die politischen Führer und Institutionen und deren Glaubwürdigkeit ab [14, 17, 18]. Vor diesem Hintergrund ist eine vollständig transparente, faktenbasierte Kommunikation von entscheidender Bedeutung.

\section{Ethische Entscheidungsfindung bei fehlenden Ressourcen}

Die ethische Entscheidungsfindung bei Katastrophen erfordert per Definition einen spezifischen Ansatz, insbesondere wenn ein großes Ungleichgewicht zwischen Ressourcenverfügbarkeit und Ressourcenbedarf besteht [8, 19-21]. In einer solchen Situation basieren Entscheidungen typischerweise auf Kontextparametern (Sicherheit, Zugänglichkeit, Verfügbarkeit und Fähigkeit) sowie den erwarteten patientenindividuellen $\mathrm{Be}$ handlungsergebnissen [14]. Es ist von grundlegender Bedeutung, dass alle $\mathrm{Pa}$ tienten so lange wie möglich nach den besten Standards versorgt werden. Sobald dies jedoch nicht mehr garantiert werden kann, soll die Priorisierung alle Patienten umfassen, die Ressourcen benötigen, unabhängig davon, ob sie als direkte Folge der Katastrophe oder aus einem anderen Grund krank oder verletzt wurden.

Die Anfangsphase der aktuellen Pandemie hat gezeigt, dass die Spitzenkapazität eines jeden Gesundheitssystems zu einem bestimmten Zeitpunkt überfordert sein kann, was möglicherweise $\mathrm{zu}$ einem echten Mangel an Intensivbetten, Beatmungsgeräten, PSA und der gesamten Wiederbelebungskapazität führt. Wenn dies eintritt, müssen Entscheidungen hinsichtlich der Ressourcenzuweisung getroffen werden. Solche Entscheidungen sollen zeitnah (nicht vorbeugend, aber nicht zu spät) und konsistent getroffen werden. Wie oben erwähnt, sollen diese Entscheidungen nicht nur von einzelnen Institutionen oder Angehörigen von Gesundheitsberufen getroffen werden, sondern auf Protokollen auf Systemebene basieren. Auf verschiedenen operativen Ebenen sollen „Ethikteams“ eingerichtet werden, um einzelne Angehörige von Gesundheitsberufen bei der Verantwortung für Rationierungsentscheidungen zu unterstützen und/oder sie zu entlasten [22, 23]. Sobald Entscheidungen getroffen wurden, soll das medizinische Personal entsprechend handeln, und die, die den definierten ethischen Rahmen nicht akzeptieren können, sollen vorzugsweise klinische Unterstützungsfunktionen in Bereichen übernehmen, 
in denen keine Rationierungsentscheidungen erforderlich sind. Solche Verteilungsentscheidungen sollen jederzeit gut dokumentiert sein (idealerweise auch in einem Register), um Transparenz und zukünftige Nachprüfungen zu ermöglichen.

Die ethische Entscheidungsfindung im Kontext einer Pandemie ist komplex. Sie soll auf der sorgfältigen Anerkennung verschiedener, manchmal widersprüchlicher, ethischer Grundsätze und gesellschaftlicher Präferenzen im konkreten Kontext der Ressourcenverfügbarkeit und der Bedürfnisse zum jeweiligen Zeitpunkt beruhen [2, 13]. Obwohl wir die Tatsache anerkennen, dass es keine universelle „Wahrheit“ gibt, möchte die ERC-Ethik-Arbeitsgruppe einige Überlegungen hervorheben, um die Gesundheitssysteme bei der Entwicklung ihrer lokalen Leitlinien $\mathrm{zu}$ informieren:

- Wenn es wirklich ein Ungleichge-

wicht zwischen den verfügbaren und benötigten Ressourcen gibt, würden die meisten Autoren für ein gewisses $\mathrm{Maß}$ an „Verteilungsgerechtigkeit“ eintreten, was „das größte Gut für die größte Anzahl von Menschen“ bedeutet und die Bedürfnisse der Gesellschaft höher bewertet als die eines Individuums [8, 12, 19, 24].

- Dieses Konzept ist in der Praxis äußerst schwierig anzuwenden. Ein in erster Linie auf „Wohlergehen“ basierter Ansatz mag im Kontext einer Katastrophe vernünftig sein, aber es ist schwierig zu definieren, was tatsächlich als Wohlergehen gilt und wie es wirklich maximiert werden kann. Dies beinhaltet potenzielle Konflikte zwischen Quantität und Qualität der Lebensjahre sowie die Herausforderung, die Lebensqualität $\mathrm{zu}$ bewerten und vorherzusagen.

- Behandlungsteams sollen für jeden einzelnen Patienten sorgfältig dessen Überlebenschancen und/oder "gutes" langfristiges Outcome sowie den erwarteten Ressourcenverbrauch bewerten. Da es sich hierbei nicht um statische Fakten handelt, soll eine solche Bewertung regelmäßig überprüft werden. Wir sind der Meinung, dass es in diesem speziellen Kontext keinen ethischen Unterschied zwischen dem Zurückhalten oder der Beendigung von medizinischer Behandlung gibt, selbst wenn das eine passiv und das andere aktiv erfolgt. Wir erkennen zwar an, dass die Standpunkte je nach kulturellem und ethischem Hintergrund unterschiedlich sein können, sind jedoch der Ansicht, dass der Entzug medizinischer Unterstützung sich ethisch von einer aktiven Beendigung des Lebens unterscheidet, die wir selbst während einer Pandemie als ethisch nicht zulässig erachten [25, 26]. Eine angemessene Komfortpflege am Lebensende ist in jedem Fall obligatorisch.

- Statt sich ausschließlich auf Expertenmeinungen zu berufen, sollen die wenigen Erkenntnisse, die es in der Literatur gibt, sorgfältig bedacht werden.

- Es gibt keine ethischen Gründe, bestimmte Gruppen aufgrund von Beruf, Rang, Status oder ähnlichen Kriterien zu bevorzugen. Auch persönliche Merkmale von Menschen wie Zahlungsfähigkeit, Lebensstil oder Verdienste um die Gesellschaft sollen bei der Priorisierung nicht als ethische Kriterien herangezogen werden. Einige Autoren befürworten die Priorisierung von medizinischem Personal und anderen „kritischen Berufen" aufgrund ihres (schwer zu ersetzenden) ,instrumentellen Werts" und der Risiken, die sie bereitwillig eingehen $[2,23]$. Diese Argumentation wäre jedoch nur relevant, wenn die identifizierten Personen tatsächlich „Schlüsselrollen“ spielen, was oft schwierig zu definieren ist, und wenn ein langfristiger Mangel bei dieser Art der „Schlüsselrolle“ zu erwarten ist [13]. Wir sind der Meinung, dass kategoriale Einschlüsse (wie im obigen Beispiel) oder Ausschlüsse (schwere chronische Lungenerkrankungen, schwere kognitive Beeinträchtigungen usw.) ethisch fehlerhaft sind [4, 23]. Innerhalb der ethischen Grenzen von „autonomy“, „beneficence and nonmaleficence“ (Selbstbestimmung, Fürsorge und Schadensvermeidung) ist jedes Leben es wert, gerettet zu werden. Anstatt Bevölkerungsgruppen zu identifizieren, für die es nicht mehr erforderlich ist, ihre „Berechtigung“ zum Erhalt bestimmter Ressourcen zu bewerten, wenn diese Ressourcen knapp sind, erfordern die ethischen Grundsätze von Gerechtigkeit und Gleichheit eine unvoreingenommene Bewertung jedes einzelnen Patienten.

- Wenn Patienten wirklich gleich sein sollten, würden sich einige immer noch auf das Prinzip „Wer zuerst kommt, mahlt zuerst" verlassen. Andere sind jedoch der Meinung, dass dies zu Ungerechtigkeit führt, beispielsweise, wenn Personen später in der Pandemie krank werden, weil sie sich strikter an empfohlene Maßnahmen im Bereich der öffentlichen Gesundheit halten, oder wenn Personen aufgrund sozialer Ungleichheit schlechteren Zugang zur Gesundheitsversorgung haben, und würden unter diesen Umständen einen egalitäreren Ansatz befürworten (z. B. durch ein Losverfahren; [2, 23]). Eine Möglichkeit, mit diesem Dilemma umzugehen, besteht darin, innerhalb des vorgegebenen ethischen Rahmens die Unterscheidung zwischen Einzelfällen zu optimieren, indem beispielsweise nicht nur ihr Ausgangsstatus, sondern auch ihre Entwicklung und ihr Ansprechen auf die Behandlung berücksichtigt werden.

- Die Kriterien sind nicht statisch und müssen rechtzeitig an Änderungen der COVID-19-Behandlungsmöglichkeiten, der Epidemiologie und der Krankenhausressourcen angepasst werden [4].

Jede Entscheidung in Bezug auf die Einschränkung der Behandlung zu jedem Zeitpunkt im Verlauf der Versorgung muss dem Patienten und seinen Angehörigen respektvoll und einfühlsam, mit voller Transparenz und Nennung der Direktive mitgeteilt werden. Dem Patientenkomfort soll jederzeit die gebührende Aufmerksamkeit gewidmet werden. 


\section{Vorausschauende Behandlungsentscheidungen}

Vorausschauende Behandlungsentscheidungen (ACP; Patientenverfügung) sollen bei allen Patienten mit einem erhöhten Risiko für einen Kreislaufstillstand oder einem voraussehbar schlechten Ergebnis im Fall eines Kreislaufstillstands in Betracht gezogen werden. Sie sollen Entscheidungen über Wiederbelebung, mechanische Beatmung, Aufnahme auf die Intensivstation und Aufnahme in ein Krankenhaus beinhalten. Bei Patienten mit bereits bestehender ACP kann es erforderlich sein, deren Angemessenheit im gegebenen Kontext zu bewerten. Die Diskussion über ACP soll den Patienten (falls möglich), seine Angehörigen (wenn der Patient zustimmt), seinen behandelnden Arzt und andere beteiligte Angehörige der Gesundheitsberufe (z. B. Intensivärzte, Krankenschwestern, Palliativteam) einbeziehen. Wir sind uns bewusst, dass dies im Zusammenhang mit sozialer Distanzierung, bei der viel Kommunikation über Telefon oder Videoverbindung erfolgt, eine Herausforderung darstellen kann [27, 28]. Darüber hinaus bestehen noch wichtige Wissenslücken, die die Prognosestellung im Kontext mit COVID-19 erschweren.

\section{Hinweise zum Zurückhalten und Beenden der Reanimation}

Die allgemeinen Grundsätze der Ethik in Notfällen und bei der Wiederbelebung bleiben während der COVID-19-Pandemie gültig $[1,12]$. Die kardiopulmonale Wiederbelebung (CPR) soll als „,bedingte" Behandlung betrachtet werden, und die Gesundheitssysteme sollen Kriterien für die Entscheidungsfindung über die Wiederbelebung unter Berücksichtigung ihres spezifischen lokalen Kontextes implementieren, rechtlich, kulturell und organisatorisch. Die Wiederbelebung soll nicht begonnen oder fortgesetzt werden, wenn die Sicherheit des Helfers nicht ausreichend gewährleistet werden kann, wenn eine offensichtliche tödliche Verletzung oder der Tod vorliegen oder wenn es eine gültige und relevante $\mathrm{Pa}$ tientenverfügung gibt (s. ,... Sicherheit des Helfers" unten).
Das Outcome bei einem nicht defibrillierbaren Kreislaufstillstand durch Hypoxämie aufgrund einer COVID-19Pneumonie ist sehr schlecht [17, 29]. In einem solchen Fall können Gesundheitssysteme (und/oder Helfer) davon ausgehen, dass das Risiko eines Schadens den erwarteten Nutzen einer Wiederbelebung übersteigt; dies stellt einen Grund für die frühzeitige Beendigung der Wiederbelebung dar.

\section{Änderungen der Reanimation zur Sicherheit des Helfers}

Die Sicherheit der Helfer - sowohl von Laienhelfern als auch medizinischem Fachpersonal - ist wichtig. Bei der Wiederbelebung kommt es zwangsläufig zu einem Abwägen zwischen dem Risiko für den Helfer und dem Nutzen für den Patienten. Während medizinisches Personal versucht, das Risiko für sich so niedrig wie möglich $\mathrm{zu}$ halten, akzeptiert es routinemäßig ein bestimmtes Risiko als Teil seines Berufs. Bis zu einem gewissen Grad gilt dies auch für Laien und wird bei diesen von ihrer Beziehung zum Opfer sowie von ihrer Risikowahrnehmung abhängen. Das Schlüsselproblem bei der Wiederbelebung während der COVID19-Pandemie besteht darin, dass sowohl das genaue Risiko für den Anbieter als auch der wahre Nutzen für den Patienten unbekannt sind.

Viele Angehörige der Gesundheitsberufe sehen sich unabhängig vom Risiko verpflichtet, den Patienten zu behandeln und nach besten Kräften zu helfen. Für Ärzte spiegelt sich dies im hippokratischen Eid wider. Während sie ihr Bestes für einen einzelnen Patienten geben, sollen sich Angehörige von Gesundheitsberufen auch ihrer Verantwortung gegenüber ihren Verwandten, Kollegen und der Gesellschaft bewusst sein [4]. Angehörige von Gesundheitsberufen, die das Risiko einer Übertragung unterschätzen, können Viren auf den Rest ihres Teams und innerhalb der Gesellschaft verbreiten und das Gesundheitssystem weiter belasten [30, 31].

CPR birgt ein klares Risiko für die Übertragung von Infektionskrankheiten, selbst wenn es sich dabei nur um CPR mit Thoraxkompression handelt $[11,32]$.
Medizinisches Personal soll daher in allen Fällen mit bestätigtem oder vermutetem COVID-19 geeignete PSA verwenden (und über deren ordnungsgemäße Verwendung informiert sein). Die Art der PSA wird im einleitenden Teil dieser Leitlinien definiert. Laien oder Ersthelfer sollen sich so weit wie praktisch möglich schützen und Aktionen mit einem hohen Übertragungsrisiko meiden, insbesondere wenn sie selbst ein großes Risiko für ein schlechtes Outcome im Fall einer Übertragung haben (ältere Menschen, chronische Lungenerkrankungen, Herzerkrankungen).

Helfer, die Pflegekräfte oder Haushaltsmitglieder des Opfers sind, wurden möglicherweise bereits exponiert und sind wahrscheinlich eher bereit, CPR durchzuführen, unabhängig vom potenziell erhöhten Risiko.

In der gegenwärtigen Situation ist es sehr wichtig, nach jedem Wiederbelebungsversuch systematisch eine Nachbesprechung durchzuführen und die Teamleistung, den medizinischen und ethischen Entscheidungsprozess sowie mögliche Probleme wie den persönlichen Schutz und die Sicherheit der Rettungskräfte zu adressieren.

\section{Verantwortlichkeit ein- zelner Angehöriger der Gesundheitsberufe}

Trotz des erheblichen Stresses, der durch die aktuelle Pandemie verursacht wird, sollen Angehörige der Gesundheitsberufe

- nach besten Kräften helfen,

- ihr Handeln an den vorliegenden Leitlinien ausrichten,

- sich, ihre Patienten und ihre Kollegen vor einer Übertragung schützen,

- Ressourcen verantwortungsvoll einsetzen, z. B. die Verschwendung oder unangemessene Verwendung von Schutzausrüstung vermeiden,

- medizinische (ethische) Entscheidungen ordnungsgemäß dokumentieren und kommunizieren,

- auch für Patienten mit akuten oder chronischen Problemen, die nicht in direktem Zusammenhang mit COVID-19 stehen, eine kontinuierliche Versorgung gewährleisten, 
- mitfühlend und einfühlsam gegenüber den emotionalen und psychologischen Bedürfnissen von Kollegen sowie Patienten und deren Angehörigen sein. Erwägen Sie ggf. eine Überweisung und Nachkontrolle.

\section{Korrespondenzadresse}

\section{K. G. Monsieurs}

European Resuscitation Council VZW

Emile Vanderveldelaan 35, 2845 Niel, Belgien dirks@grc-org.de

\section{Einhaltung ethischer Richtlinien}

Interessenkonflikt. P. Van de Voorde, L. Bossaert, S. Mentzelopoulos, M.T. Blom, K. Couper, J. Djakow, P. Druwé, G. Lilja, I. Lulic, V. Raffay, G.D. Perkins und K.G. Monsieurs geben an, dass kein Interessenkonflikt besteht.

Für diesen Beitrag wurden von den Autoren keine Studien an Menschen oder Tieren durchgeführt. Für die aufgeführten Studien gelten die jeweils dort angegebenen ethischen Richtlinien.

\section{Literatur}

1. Bossaert LL, Perkins GD, Askitopoulou Hetal (2015) European Resuscitation Council Guidelines for Resuscitation 2015: Abschnitt 11. The ethics of resuscitation and end-of-life decisions. Resuscitation 95:302-311

2. Emanuel EJ, Persad G, Upshur R et al (2020) Fair allocation of scarce medical resources in the time of COVID-19. N Engl J Med. https://doi.org/10.1056/ NEJMsb2005114

3. Gostin LO, Friedman EA, Wetter SA (2020) Responding to COVID-19: how to navigate a public health emergency legally and ethically. Hastings Cent Rep. https://doi.org/10.1002/hast.1090

4. Chan PS, Berg RA, Nadkarni VM (2020) Code blue during the COVID-19 pandemic. Circ Cardiovasc Qual Outcomes. https://doi.org/10.1161/ CIRCOUTCOMES.120.006779

5. Lazzerini M, Putoto G (2020) COVID-19 in Italy: momentous decisions and many uncertainties. Lancet. https://doi.org/10.1016/S2214109X(20)30110-8

6. Couper K, Taylor-Phillips S, Grove A, Freeman K, Osokogu O, Court R, Mehrabian A, Morley PT, Nolan JP, Soar J, Perkins GD (2020) COVID-19 in cardiac arrest and infection risk to rescuers: a systematic review. Resuscitation.https://doi.org/ 10.1016/j.resuscitation.2020.04.022

7. Resuscitation council UK statements; url: https://www.resus.org.uk/media/statements/ resuscitation-council-uk-statements-on-covid19-coronavirus-cpr-and-resuscitation/; accessed 05 April 2020

8. Biddison LD, Berkowitz KA, Courtney B et al (2014) Ethical considerations: care of the critically ill and injured during pandemics and disas- ters: CHEST consensus statement. Chest $146(4$ Suppl):e145S-e155S

9. Disaster bioethics; url: https://disasterbioethics. com/covid-19/; accessed 05 April 2020

10. Edelson DP, Sasson C, Chan PS et al (2020) Interim Guidance for Basic and Advanced Life Support in Adults, Children, and Neonates With Suspected or Confirmed COVID-19: From the Emergency Cardiovascular Care Committee and Get With the Guidelines ${ }^{\circledR}$-Resuscitation Adult and Pediatric Task Forces of the American Heart Association in Collaboration with the American Academy of Pediatrics, American Association for Respiratory Care, American College of Emergency Physicians, The Society of Critical Care Anesthesiologists, and American Society of Anesthesiologists: Supporting Organizations: American Association of Critical Care Nurses and National EMS Physicians [published online im Druck, 2020 Apr 9. Circulation. https://doi.org/10.1161/CIRCULATIONAHA.120. 047463

11. Denis et al (2020) Transdisciplinary insights - Livin Paper Rega Institute Leuven Belgium. https://rega. kuleuven.be/if/corona_covid-19. Zugegriffen: 6 . Apr. 2020

12. Ethical guidance Belgian Resuscitation Council, Belgian Society of Emergency and Disaster Medicine; url: https://www.besedim.be/wp-content/ uploads/2020/03/Ethical-decisionmaking-inemergencies_COVID19_22032020_final-1.pdf; accessed 05 April 2020

13. Kim SYH, Grady C (2020) Ethics in the time of COVID: What remains the same and what is different. Neurology. https://doi.org/10.1212/ WNL.0000000000009520

14. Koonin LM, Pillai S, Kahn EB, Moulia D, Patel A (2020) Strategies to inform allocation of stockpiled ventilators to Healthcare facilities during a pandemic. Health Secur. https://doi.org/10.1089/hs. 2020.0028

15. Schiariti V (2020) The human rights of children with disabilities during health emergencies: the challenge of COVID-19. Dev Med Child Neurol. https://doi.org/10.1111/dmcn.14526

16. Lewnard JA, Lo NC (2020) Scientific and ethical basis for social-distancing interventions against COVID-19. Lancet Infect Dis. https://doi.org/10. 1016/S1473-3099(20)30190-0

17. Fritz Z, Perkins GD (2020) Cardiopulmonary resuscitation after hospital admission with covid19. BMJ 369:m1387. https://doi.org/10.1136/bmj. m1387

18. Legido-Quigley H, Asgari N, Teo YY et al (2020) Are high-performing health systems resilient against the COVID-19 epidemic? Lancet 395(10227):848-850. https://doi.org/10.1016/ S0140-6736(20)30551-1

19. Satkoske VB, Kappel DA, Disaster Ethics DVMA (2019) Shifting priorities in an unstable and dangerous environment. Crit Care Clin 35(4):717-725 https://doi.org/10.1016/j.ccc.2019.06.006

20. Somes J, Donatelli NS (2014) Ethics and disasters involving geriatric patients. J Emerg Nurs 40(5):493-496. https://doi.org/10.1016/j.jen. 2014.05.013

21. Mezinska S, Kakuk P, Mijaljica G, Waligóra M, O'Mathúna DP (2016) Research in disaster settings: a systematic qualitative review of ethical guidelines. BMC Med Ethics 17(1):62. https://doi. org/10.1186/s12910-016-0148-7

22. Arie S (2020) COVID-19: can France's ethical support units help doctors make challenging decisions? BMJ 369(2020):m1291-2. https://doi. org/10.1136/bmj.m1291
23. White DB, Lo B (2020) A framework for rationing ventilators and critical care beds during the COVID 19 pandemic. JAMA. https://doi.org/10.1001/ jama.2020.5046

24. Merin O, Miskin IN, Lin G, Wiser I, Kreiss Y (2011) Triage in mass-casualty events: the Haitian experience. Prehosp Disaster med 26(5):386-390. https://doi.org/10.1017/S1049023X11006856

25. Mentzelopoulos SD, Slowther AM, Fritz Z et al (2018) Ethical challenges in resuscitation. Intensive Care Med 44(6):703-716. https://doi.org/10.1007/ s00134-018-5202-0

26. Sprung CL, Ricou B, Hartog CS et al (2019) Changes in end-of-life practices in European intensive care units from 1999 to 2016 [published correction appears in JAMA. 2019 Nov 5;322(17):1718. JAMA 322(17):1-12.https://doi.org/10.1001/jama.2019. 14608

27. Boettcher I, Turner R, Briggs L (2015) Telephonic advance care planning facilitated by health plan case managers. Palliat Support Care 13(3):795-800

28. Tieu C, Chaudhry R, Schroeder DR, Bock FA, Hanson GJ, Tung EE (2017) Utilization of patient electronic messaging to promote advance care planning in the primary care setting. Am J Hosp PalliatCare 34(7):665-670

29. Shao F, Xu S, Ma X, Xu Z, Lyu J, Ng M, Cui H, Yu C, Zhang Q, Sun P, Tang Z (2020) In-hospital cardiac arrest outcomes among patients with COVID19 pneumonia in Wuhan, China. Resuscitation. https://doi.org/10.1016/j.resuscitation.2020.04. 005

30. Ofner-Agostini M, Gravel D, McDonald LC et al (2006) Cluster of cases of severe acute respiratory syndromeamong Torontohealthcareworkersafter implementation of infection control precautions: a case series. Infect Control Hosp Epidemiol 27(5):473-478

31. Marineli F, Tsoucalas G, Karamanou M, Androutsos G (2013) Mary Mallon (1869-1938) and the history of typhoid fever. Ann Gastroenterol 26(2):132-134

32. Ott M, Krohn A, Jaki C, Schilling T, Heymer J (2020) CPR and COVID-19: aerosol-spread during chest compressions. Zenodo. https://doi.org/10.5281/ zenodo.3739498 